\title{
Die Zeitform des Schweigens
}

\author{
Flexionen der (auto)biographischen Frage am Beispiel \\ William Wolfensbergers
}

Franzisca Pilgram-Frühauf

Angenommen, es gäbe die biographische Frage, die hinter jeder Lebensbeschreibung stünde, dann lautete sie für die folgende Skizze: "Wer war William Wolfensberger?" Das Präteritum als Tempus des Satzes deutet auf einen abgeschlossenen, objektiv fassbaren Zeitraum zwischen 1889 und 1918, der Lebenszeit des biographischen Subjekts. Der Eigenname "William Wolfensberger" bestärkt dies wie ein aufgedrückter Fingerabdruck als "signum authenticum», Zeichen der Identität, wie sie im Rahmen sozialer und rechtlicher Ordnungen von einem Individuum abverlangt wird und für jeden anderen Namen ebenso gilt. Das Interrogativpronomen schliesslich leitet - mit entsprechenden Satzzeichen - eine Ergänzungsfrage ein. Diese steuert mit einem gewissen Nachdruck darauf hin herauszufinden, was sich hinter dem Präteritum und hinter dem Namen verbirgt. Sie sucht nach der Zeiteinheit eines Lebens und gibt Anstoss zu deren Beschreibung. Mögliche Antworten wären dem Zivilstandsregister, einer möglichst scharfen Photographie, einem offiziellen Curriculum vitae oder einer ausführlichen biographischen Darstellung zu entnehmen.

Der eingeschlagene Weg führt zu einem relativ geschlossenen, in sich stimmigen Gesamtbild der untersuchten Zeitspanne, in der das leibhaftige Individuum ins Leben tritt und wieder aus ihm scheidet; allerdings auf Kosten der Komplexität des Begriffs der Lebenszeit. Denn vermag eine Biographie festzuhalten, wer ein Mensch war - sei es, dass dieser noch lebt oder wie Wolfensberger vor beinahe einem Jahrhundert gestorben ist? Gelingt es, nach minuziösem Nachforschen, zu ergründen, von welcher Wirklichkeit er umgeben war, wie er dieser Wirklichkeit als Wirklicher begegnet ist und welche Wirkungen - zumeist im Verborgenen - von ihm ausgegangen sind und sich über seinen Tod hinaus erstreckten? Gibt es so etwas wie ein tatsächliches Leben, das man nur unter den Trümmern der Zeit freizulegen braucht?

Dass der Gattung der Biographie lange Zeit mit grosser Skepsis begegnet wurde und dass die "biographische Illusion" (Pierre Bourdieu) ein gängiges Schlüsselwort geworden ist, gebietet methodische Vorsicht. Daher muss die biographische Frage 
- im Anschluss an ein paar grundsätzliche Überlegungen - auf ihre Flexionen hin überprüft, reflektiert werden: Wie verhält sich das Unternehmen der (Auto)Biographie, interessiert am Präteritum der Lebenszusammenhänge, zur präsentischen Erfahrung, dass das Leben im Vollzug als Fragment vorbeizieht, und zur offenen Frage, was im Leben oder gar darüber hinaus, jedenfalls in der Zukunft, noch auf einen zukommt?

\section{Biographie vs. Biographeme}

Inzwischen entspricht die These von der "Fiktion des Faktischen" (Hayden White) auch in der Biographie-Forschung einem allgemeinen Konsens und man ist an einem Punkt angekommen, an dem man die unvermeidliche Fiktionalität von Biographien nicht mehr als bedauerliches Manko beklagt. Der lange Zeit suspekten Arbeitsmethode wird vielmehr zugestanden, insbesondere im interdisziplinären Dialog erhebliche Erkenntnisgewinne zu erzielen - allerdings nur unter gewissen Vorsichtsmassnahmen, die es zu berücksichtigen gilt. Als Ausgangspunkt bieten sich zwei Begriffe an, die das notwendige Umdenken in der Biographieforschung konzise zum Ausdruck bringen: Diltheys zentrales biographisches Postulat der Naturbeschreibung einer "psycho-physischen Lebenseinheit" und Barthes Reduktionsfigur der "Biographeme».

Wilhelm Dilthey definierte 1883 die Biographie als Erfassung der ganzen Wirklichkeit eines Individualdaseins. Zwar erkannte er, dass die Arbeit des Biographen stets auf materiellen Quellen, so genannten "Resten", beruht, die immer auch unvollständig und begrenzt sind und das grundsätzliche hermeneutische Problem der Bedeutung und des Deutens aufwerfen. Der Weg, den Dilthey einschlug, ist aber erklärtermassen eine (geisteswissenschaftliche) Lösung dieses Problems: die Verwandlung von "Resten" qua Verstehen in einen umfassenden Wirkungszusammenhang. Der Begriff des Zusammenhangs bildet die Leitfigur seiner Biographik, auch auf einer formalen Ebene. Nach Dilthey scheint eine biographische Wahrheit um so überzeugender gewonnen, je mehr die narrative Wiedergabe eine innere Kohärenz und Kausalstruktur aufweist. Im Hintergrund steht das alte Genre "Lebenslauf", bei dem Chronos das Leben verwaltet, verbunden mit der Annahme, dass eine Kohärenz des Individuums besteht, die sich in einer entsprechend geordneten Narration in zeitlicher Folgerichtigkeit wiedergeben und schlüssig abbilden lässt. Die Erzählung folgt entlang einer lebenszeitlichen Geraden, die in der 
Geburt ihren Anfang nimmt und mit dem Tod endet, und erlaubt es, die fremde Person quasi leibhaftig zu begreifen.

Es war Roland Barthes, der mit seinem Konzept der "Biographeme" die wohl schärfste Opposition zu jenem Ideal formuliert hat, das seit Dilthey teilweise bis heute gültig ist. Wenn ein Autor tot ist, vermag keine Rekonstruktion der Lebenszeit diesen wieder zum Leben zu erwecken. Vielmehr forderte Barthes, anstatt Kohärenz zu stiften, die Vorstellung einer schicksalhaften Abgeschlossenheit aufzugeben: »[W]äre ich Schriftsteller und tot, wie sehr würde ich mich freuen, wenn mein Leben sich dank eines freundlichen und unbekümmerten Biographen auf ein paar Details, einige Vorlieben und Neigungen, sagen wir auf `Biographeme`, reduzieren würde, deren Besonderheit und Mobilität ausserhalb jeden Schicksals stünde.» (Sade Fourier Loyola, 1971) Das Detail also, besser noch: die Details, aus dem Curriculum herausgebrochene Einzelphänomene, machen hier Methode, nicht die zusammenhängende Lebensgeschichte. Dies hat auch Folgen für die narrative Struktur: Anstatt Schweigen beredt $\mathrm{zu}$ füllen oder auch stillschweigend $\mathrm{zu}$ verschwiegen, was den lebenszeitlichen Zusammenhang gefährdet, nimmt sie nun auch Inkohärentes und Augenblickshaftes auf, enthält Brüche und Leerstellen. Diese dienen nicht als Einladung, das Unganze der Texte durch Interpretation doch noch ganz $\mathrm{zu}$ machen oder durch die Lücken und Ritzen hindurch voyeuristisch zu enthüllen, was sich dahinter verbirgt. Sie legen vielmehr offen, dass gegen die Annahme, ein Leben beschreiben zu können, erst einmal wahrgenommen werden muss, dass es sich nicht um das Leben selbst handelt, sondern um Erschreibungen dieses Lebens. Im Fall von Wolfensberger bedeutet dies: Die biographische Frage ist jenseits der Deutungen verschiedenster Art, von denen er vor allem nach seinem Tod umstellt war, und aus seiner eigenen Hinterlassenschaft nicht zu beantworten.

\section{Beschreibungen - Erschreibungen}

Meine Vermutung lautet, dass das Schweigen angesichts der biographischen Grundfrage einer bestimmten Flexionsweise entspringt: Was verändert sich, wenn die im Präteritum gestellte Frage um das Spektrum der Gegenwart und der Zukunft erweitert und relativ zum "Jetzt" des Schreibens in der Zeitform verschoben wird: Wer war, wer ist, wer wird $\mathrm{x}$ sein? 


\section{a. Wer war $x$ ?}

Gemäss den Biographien, die meist kurz nach Wolfensbergers Tod entstanden sind (besonders ausführlich diejenige von Max Konzelmann, 1924), gehören folgende Stationen zu dessen Vita:

William Wolfensberger wurde am 17. Juni 1889 als drittjüngstes Kind einer grossen Kaufmannsfamilie in Zürich-Hottingen geboren. Aufgrund einer körperlichen Schwäche und eines sensiblen Geistes war er für den kaufmännischen Beruf, für den er aufgrund der Familientradition vorgesehen war, wenig geeignet und trat 1904 in die zweite Klasse des kantonalen Gymnasiums über - wohl bereits mit dem Berufswunsch im Hintergrund, Pfarrer zu werden. In einer inneren Krise jedoch zerbrachen die religiösen Überzeugungen und die beruflichen Pläne. Im Herbst 1909, nach bestandener Maturität, schrieb sich Wolfensberger als Germanistikstudent an der Universität Zürich ein. In diesem Gebiet hoffte er, seine Begeisterung für die Literatur und seine Vorstellung von einem der Kunst geweihten Leben am ehesten nähren zu können. Er wurde enttäuscht. Linguistische Studien und die schulmeisterliche, philologische Behandlung der Literatur vermochten ihn nicht $\mathrm{zu}$ befriedigen. Vor allem aber liess ihn seine frühere Neigung zum Pfarramt und zur lebendigen Religiosität nicht los, so dass er am Ende seines ersten und einzigen germanistischen Semesters an die Theologische Fakultät wechselte. Der feste Entschluss, nun doch noch Pfarrer zu werden, geschah allerdings ohne das väterliche Einvernehmen. Die immer schon angespannte Beziehung zum Vater eskalierte mit der Folge, dass der Student auf die Strasse gestellt wurde und selbst für ein Zimmer und finanzielles Auskommen sorgen musste. In die Zeit des Suchens und Ringens nach dem richtigen Weg fiel ausserdem die schicksalhafte Begegnung mit einer Frau, welcher Wolfensberger leidenschaftliche Liebe entgegenbrachte, ohne aber Erwiderung zu finden. Diese unerfüllte Liebe und der Verlust des Elternhauses prägten Wolfensbergers Leben fortan wesentlich.

Zum zweifachen Leid kam ein drittes hinzu. Der ordinierte Pfarrer fand im abgeschiedenen Münstertal, in den oberen Dörfern Fuldera, Tschierv und Lü, eine erste Pfarrstelle und trat sie im Frühling 1914 voller Hoffnung an, durch die Arbeit für andere "das Schwere in ihm« überwinden zu können. Als der Erste Weltkrieg ausbrach, übte er neben seinen Verpflichtungen als Pfarrer auch noch das Amt des Gemeindepräsidenten und Kassiers aus, wog Mehl, Polenta, Petrol ab und verteilte es billig unter die Bevölkerung, verkaufte Holz aus dem Gemeindewald, überwachte den Bau der Wasserleitung und setzte sich für den Umbau des verwahrlosten Friedhofs ein, indem er durch 
zahlreiche Vorträge und Werbeblätter die Finanzierung sicher stellte. Zeitweilig übernahm Wolfensberger auch den Unterricht an der Acht-Klassen-Schule mit dreissig Wochenstunden. Die Gemeinde dankte ihm den selbstlosen Einsatz mit dem Ehrenbürgerrecht. Das Verhältnis zu ihrem Pfarrer verschlechterte sich allerdings, als er der Gemeindeversammlung einen Plan zur Sanierung der Finanzen vorlegte. Sein Vorschlag (eine Form von progressiver Besteuerung) schürte den Hass einiger begüterter Bürger, bis Wolfensberger Ende September 1916 unter dem Druck der Gegner nur noch die Kündigung blieb. Zum Verlust des Elternhauses und zur unerfüllten Liebschaft kam das dritte Leid der verlorenen Gemeinde hinzu.

Krank und bitter enttäuscht, aber dennoch froh, nach einer Zeit der Untätigkeit und Ungewissheit wieder arbeiten zu können, trat Wolfensberger Ende April 1917 seine neue Stelle in Rheineck an. Bald ging es ihm besser. Das offene Vertrauen, das ihm die Kinder entgegenbrachten, und eine tiefe Verbundenheit mit einzelnen Menschen, trugen ihn über die Enttäuschungen hinweg. Obwohl alte Wunden aus der Zeit in Fuldera erneut aufbrachen, stürzte er sich voller Liebe und Hingabe in die Arbeit an seiner Gemeinde, besuchte während der Grippeepidemie, von der Rheineck besonders stark betroffen war, furchtlos Kranke und Sterbende. Wolfensberger selber, der schon seit einiger Zeit von "Ahnungen eines frühen Todes« erfüllt war, erkrankte dreimal an der heimtückischen Seuche und erlag ihr in den Morgenstunden des 6. Dezember 1918 - mitten in seinem dreissigsten Lebensjahr.

So viel gescheiterte Hoffnungen, Fehlschläge und Erniedrigungen die kurze Lebensspanne Wolfensbergers enthält, so stark ist in den verschiedenen Darstellungen auch ein Bestreben der Biographen zu spüren, dieses früh abgebrochene und in Leid und Schmerzen zerbrochene Leben als ganzes erscheinen zu lassen und heil zu machen: Trotz seines frühen Todes sei Wolfensberger ein "Frühvollendeter». Wenn ihm auch viel Schweres widerfahren sei, er habe sich in sein Schicksal gefügt und sein Leiden letztlich überwunden.

Um dies nachzuvollziehen, verwenden die Biographen verschiedene Modelle der Erlösung. Eines davon liegt, geprägt durch die christliche Leidensthematik, im Glauben begründet:Wolfensbergers religiöser und theologischer Hintergrund, den er ganz für die Verkündigung und die Seelsorge im alltäglichen Leben der Menschen, vor allem der Armen und Hilfsbedürftigen, eingesetzt habe, sei ihm letztlich selbst als Trost zugute gekommen. Er sei über die "Schule des Leidens" einen schweren Lebensweg gegangen, aber dieser dornenreiche Weg habe ihn schliesslich zu Gott geführt, wo 
Grauen und Zweifel in Gewissheit aufgehen. Seine Gedichte, »im Predigttone gehaltene Bekenntnisse seines Glaubens", zeugten davon - und wirkten als Zeugnisse weiter, tröstend auf die Leser. Denn es liege in ihnen die Verheissung verborgen, dass im Tod Friede und Harmonie erfahren werden, welche das Leben nur augenblickshaft in der Kunst, nie aber vollkommen geben könne.

Man bleibt, mit einiger Distanz sowohl zum beschriebenen Zeithorizont als auch zur Entstehungszeit der Biographien, mit einer gewissen Ratlosigkeit oder gar Befangenheit zurück. Wie soll man auf die dargestellte Leidenstiefe reagieren? Sympathetisch, auch mitleidend, im eigenen Leiden bestärkt, oder eher befremdet? Tatsächlich, wie könnte man es beurteilen, wo man es ja nicht miterlebt hat? Wie hat Wolfensberger selbst die biographische Frage beantwortet?

\section{b. Wer ist $\mathrm{x}$ ?}

Wolfensbergers Nachlass enthält eine etwa 340 handschriftliche Heftseiten und zweiundzwanzig Kapitel umfassende Autobiographie, in welcher der Maturand im Rückblick auf seine ersten zwanzig Lebensjahre den Versuch einer Standortbestimmung unternimmt. Der Titel deutet bereits darauf hin: Das »Curriculum vitae«, das auf die Gegenwart des Schreibens hinzielt, ist eine "Geschichte ohne Schluss".

Im Zentrum steht zunächst die Auseinandersetzung mit eigenen Fehlern und Vergehen, den so genannten »Kindersünden«. Die Funktion der Autobiographie ist es nicht, "abzurechenen", sondern durch Erzählung Wahrheiten aufzudecken, die zum Teil jahrelang verschwiegen wurden. Eines der Kapitel ist daher mit dem Motto überschrieben: "Reden wir nur davon, ihr Weisen, ob es gleich schlimm ist. Schweigen ist schlimmer. Alle verschwiegenen Wahrheiten werden giftig». (Kap. XII) Dieses Zitat aus Nietzsches "Zarathustra" führt in einer progressio ad negativum vom Schlimmen über das Schlimmere zum Schlimmsten, denn "giftig werden« bedeutet so viel wie eine Wirkkraft entfalten, die unberechenbar und zerstörerisch ist. Kann diese Steigerung als Legitimation dafür betrachtet werden, doch $\mathrm{zu}$ reden, auch wenn es, aus welchem Grund auch immer, weise erscheinen könnte, den Mund zu halten? Auf die Situation des autobiographischen Schreibens übertragen: Ermutigt dies, trotz der begrenzten Sichtweise und trotz der fehlenden Kompetenz zu urteilen und zu richten, sein Leben zu beschreiben? 
Wer so fragt, übersieht eine wichtige Nebenbedingung: (Auto)Biographisches Reden enthält Begrenzungen und Lücken und bedeutet nicht, dass es das Schweigen aufhebt. Das wird deutlich anhand eines selbstreflexiv-poetologischen Satzes gegen Schluss des Curriculums: „Ein Curriculum muss immer sprunghafter werden, je mehr es sich der Gegenwart nähert«. (Kap. XX) Diese Forderung ist im Text umgesetzt, denn die narrativen Passagen werden seltener, die einzelnen Abschnitte enthalten vor allem innere Monologe, Gedankenfragmente und aphoristische Sentenzen. Sie sind kürzer und werden mit Trennungsstrichen voneinander isoliert. Inhaltlich wie formal fehlt es an Kohärenz und erzählerischer Distanz, denn das gelebte Leben ist noch zu nah, um in sich schlüssig dargestellt, und zu bewegt, um festgehalten werden zu können. Dieser Eindruck verstärkt sich beim Lesen dadurch, dass sich die Handschrift gegen Schluss stärker vorwärts neigt, schwungvoller und flüchtiger wird. Der Text scheint in der Gegenwart auf die Zukunft hin auszufransen. Er endet mit einem Pfeil, der auf die restlichen leeren Seiten weist und mit der Anmerkung versehen ist: »(Dieser Teil ist noch zu leben)《.

Der unfertige Charakter des Curriculums wir durch einen eigentümlichen Umstand noch auf die Spitze getrieben. Offenbar hat Wolfensberger das fertige Manuskript einem Lehrer zur Durchsicht gegeben. Dieser hat mit roter Tinte Korrekturen und Kommentare eingetragen, um da und dort die Orthographie zu bereinigen oder den Ausdruck zu verbessern. Jemand, wohl Wolfensberger selbst, hat nun diese roten Einträge wieder rückgängig zu machen versucht, hat die rote Farbe schwarz überschrieben oder abgekratzt und damit das Papier beschädigt. Dadurch sind weitere Lücken, sogar Löcher im Papier, entstanden, die einen Eindruck der Fehlerfreundlichkeit vermitteln und sich gegen eine druckfertige Korrektheit des Textes wehren. Es handelt sich bei diesem Text um ein Experiment, den Versuch, die Offenheit der Lebenszeit sprachlich zu artikulieren.

\section{c. Wer wird $x$ sein?}

Während im Curriculum vitae die sich reduzierende Zeitdifferenz zwischen Schreiben und Erleben ins Bewusstsein gehoben wird, verleihen Wolfensbergers Briefe punktuell der Situation des Schreibens und der jeweiligen, immer wieder sich verändernden Gegenwart Ausdruck. Sie setzen am Punkt minimaler Distanz an, so dass die Grenzen zwischen erlebendem und schreibendem Ich verschwimmen. Umso mehr bringt dieses Fortschreiten in einer ungewissen 
Zeitfolge die Zukunft ins Spiel. Denn der offene Horizont der Zukunft, in den die Autobiographie einmündet, ist das, was die aus dem Moment heraus geschriebene Selbstdarstellung durch Briefe latent begleitet. Die Zukunft wird aber auch thematisiert, indem der Briefschreiber Erwartungen an sie richtet und Pläne zur Sprache bringt.

Die geplantenVorhaben beziehen sich vor allem aufWolfensbergers literarische Tätigkeit. Enthusiastisch schreibt er Jakob Hausheer im Brief vom 13.10.1916 von seinen künstlerischen Projekten: »Du lächelst vielleicht über soviel Pläne? Du weißt, es ist dies meine einzige Stärke. S'ist mir halt immer noch, als falle mir einmal ein Königreich in den Schoss «. Wolfensberger stellt sich beim Schreiben von Briefen des öfteren vor, wie die Freunde beim Lesen lächeln. Worüber? Über seine literarischen Ambitionen, den Eifer? Zwischen den Zukunftsentwürfen und der tatsächlichen Durchführung der Pläne zeichnet sich oft eine leerstellenartige Diskrepanz ab, die Wolfensberger selbst verwirrt und verstummen lässt.

Akut wird diese Spannung vor dem Stellenantritt im Jahr 1914. Er schreibt seinem Studienfreund, Robert Lejeune, vor dem Umzug nach Fuldera: ॥ndes gehe ich ins Münstertal und bin dankbar, Roby, wie dankbar, wenn sie mich wollen und ich arbeiten kann. Zwei Tagesreisen von Zürich! Ich habe jetzt meinen Frieden gemacht ... Ich will dankbar sein, dass ich für jene Welt arbeiten kann, die wir Gott nennen«. (30.1.1914) Eine Woche zuvor heisst es: »Mir ist, als ob etwas so Entsetzliches bevorstünde, dass man es nicht aussprechen darf ". Derselbe Absender, derselbe Adressat, dieselbe nahe Zukunft: eklatanter könnte der Stimmungswechsel nicht sein, der mithin einen Umschwung im Selbstausdruck erzeugt. Liest man die beiden Briefe als aufeinanderfolgende Segmente, die im von Wolfensberger an Lejeune adressierten Briefkorpus zueinander in Beziehung stehen, so stösst man sich an dieser Inkonsequenz, die als Leerstelle wirkt. Hervorgerufen wird sie beim Briefschreiber angesichts der Ungewissheit, der Ahnungen und Befürchtungen, was die Zukunft bringen wird. Er vermag diese noch nicht in seinen Text zu integrieren, sondern antizipiert sie als offene Perspektive in Fragen, Wünschen oder im ahnungsvollen Gebot, (noch) zu schweigen. Die offene Perspektive der Zukunft wirkt bedrohlich in die Gegenwart hinein und »durchlöchert« die einzelnen Briefsegmente mit Ungewissheit. Der elliptische Ausruf "Zwei Tagesreisen von Zürich!« und der vage Hinweis auf ein unbestimmtes »Entsetzliches« vermitteln dem Adressaten die angstvolle Erwartung angesichts des Kommenden und die Spannung zwischen Hoffnung und der Befürchtung, wieder 
einmal mit dem Schlimmsten rechnen zu müssen. Die Abbrüche in und die Brüche zwischen den Briefen entsprechen einem permanenten Schwanken zwischen Aktivität und Passivität im Umgang mit der ungewissen Zukunft. Das Aktive zeigt sich in der Hoffnung und dem Wunsch, sein Leben selbst mit etwas zu füllen, das es ganz macht. Es ist die Bitte um die Möglichkeit, seinem Leben diesen Sinn, eine Kontinuität geben zu können. Diese Erwartungshaltung schlägt aber immer wieder um in den unentrinnbaren Leidensdruck zur Passivität. Die Folge davon ist Schweigen.

\section{Hermeneutik des Schweigens}

Als These muss festgehalten werden: Jeder Lebensbeschreibung ist Schweigen eingeschrieben, denn jeder dargestellte Lebensakt war einmal Gegenwart und also mit der Brüchigkeit des Lebens konfrontiert. Das gilt für die Autobiographie genauso wie für die Biographie, denn erstere kann ebenso verzerren wie die Sicht eines/r Nachgeborenen, der/die sich mit genügend Abstand um eine Gesamtschau bemüht. Die Grundgegebenheit fällt oft nur deshalb nicht auf, weil die lineare Evidenz narrativer Sprache von einem chronologischen Zeitbegriff ausgeht, der auch unserem Lebensbegriff nur allzu sehr entspricht.

Hier ist der Moment, dem Wort nachzuspüren, mit dem es jede Biographie zu tun hat: dem Bios. Gemeint ist mit dem griechischen Wort das Leben, das man einmal und irreversibel durchläuft, mit dem man rückblickend abrechnet. Es ist strukturiert in Jahre, Monate, Tage oder auch nur Bruchteile von Sekunden, und ist dem Menschen Antrieb, etwas aus sich zu machen, es im Leben zu etwas zu bringen.

1) Hier setzt die Biographie an. Der Biograph forscht minutiös nach der Lebensgeschichte des biographischen Subjekts, sucht nach allen Belangen materieller Existenz und hält die vielen Tagesgeschäfte und Zukunftspläne fest, mit denen es seinen Kalender gefüllt hat. Die Lücken in den Quellen spielen insofern eine Rolle, als sie oft die Keimzellen bilden, denen biographische Ansätze entspringen: Zeiten der Desorientierung, des Zweifels und der Krise werden harmonisierend ausgedeutet und aus einem übergeordneten Zusammenhang heraus mit Sinn gefüllt, Inkohärenzen werden zu Gunsten der Kohärenz überschrieben. Jedes Schweigen des biographischen Subjekts kann durch das Aneignungsverfahren der Interpretation 
rednerisch widerlegt, die Transparenz des Lebens wiederhergestellt werden; man braucht dazu nur genügend Vorstellungskraft.

2) Wie die Lücken in einer Lebensbeschreibung vom Biographen gefüllt und überschrieben werden, wird die bevorstehende Lebenszeit von uns gefüllt mit Projekten und Plänen, mit denen wir uns die Zukunft ausmalen. Wolfensbergers Briefe deuten vielleicht darauf hin, dass die darin ausgesprochenen Wünsche und Pläne von einem Schweigen begleitet sind, von etwas, was sich der autobiographischen Beschreibung entzieht, weil es noch nicht beschreibbar ist. Ist die Zukunft als Horizont jeder Gegenwart vielleicht das Geheimnis, demgegenüber Schweigen geboten ist? Dem widersprechen aber die vielen Projekte und Prognosen, mit denen zukünftige Zeit schon lange im Voraus bestimmt und festgelegt ist. Auch Zukunft ist und bleibt beschreibbar, solange sie auf der Geraden liegt, die durch die Punkte der Vergangenheit über die Gegenwart hinaus gezogen wird.

3) Im Übergang von Vergangenheit und Zukunft notiert der Schreiber des autobiographischen Curriculums, dieses werde immer sprunghafter, je näher er sich schreibend der Gegenwart nähere. Und kurz darauf folgt der abrupte Hinweis: „Dieser Teil ist noch zu leben«. Dieser Übergang zum Schweigen im Vollzug autobiographischen Schreibens zeigt, dass es Momente gibt, die das eigene Reden durchlöchern oder gar abbrechen lassen. Das "signum authenticum" der Lebensbeschreibung ist weder das vervollständigte Präteritum noch die Sicherheitsvorkehrungen für die Zukunft. Entscheidend ist vielmehr die Gegenwart, als Zeit, die alle chronologischen Kohärenzen sprengt. Hier löst sich die Ordnung des Bios auf und macht einer neuen Zeitwahrnehmung Platz. Das Schweigen, in dem es sich niederschlägt, versucht nicht, die Grenze zwischen Vergangenheit und Zukunft - oder gar zwischen Zeit und Ewigkeit - zu überwinden, sondern tritt selbst in einer temporalen Grenzsituation auf.Vielleicht ist damit der Kern einer Hermeneutik des Schweigens aus einer kairologischen Perspektive erfasst.

\section{IV. »Zoographie«?}

Das Schweigen in der Biographie, welches das Unbestimmte menschlicher Existenz in die Sprache hineinträgt, betrifft auch die Theologie als Reden von Gott: Auch für sie kann sich die Antwort auf die fundamentale Frage, wie man denn über Gott reden könne, nicht allein darin erschöpfen, die Lebenszeit des Gottessohns 
zu beschreiben. Denn wie für alle Biographien gilt auch für die Biographie des Glaubens: Sie beschreibt, erschreibt und schweigt.

Die Bibel spricht christologisch nicht von Bios, sondern von Zoe als demjenigen Leben, das die göttlichen Gegenwartsmomente aufnimmt.Angesichts der Zoe bleibt die durchkonjugierte biographische Frage offen. Auch der Versuch, vielleicht auf eine "Zoographie» auszuweichen, würde scheitern, denn gelänge dies, wäre die Zoe bereits verspielt. Sie entwickelt sich in unberechenbarer Präsenz und bewirkt, dass die Momente, in denen Sprache abbricht, nicht nur als Zeiten des Defizits in den Blick kommen. Das "Losungswort", das Wolfensberger in einem Brief (12.5.1916 an Jakob Hausheer) formuliert, lautet: »Was, - Zukunftspläne? ... Still geworden, aber wirklich still, dann kannst du gestillt werden«.

- Franzisca Pilgram-Frühauf ist Assistentin am Lehrstuhl für Systematische Theologie insbesondere Hermeneutik und Fundamentaltheologie der Universität Zürich. 